\title{
Knockdown of SETDB1 inhibits breast cancer progression by miR-381-3p-related regulation
}

\author{
Milu Wu, Baohua Fan, Qijing Guo, Yan Li, Rong Chen, Nannan LV, Yinzhuo Diao and Yushuang Luo* (D)
}

\begin{abstract}
Background: SET domain bifurcated 1 (SETDB1) has been widely considered as an oncogene playing a critical role in many human cancers, including breast cancer. Nevertheless, the molecular mechanism by which SETDB1 regulates breast cancer tumorigenesis is still unknown.

Methods: qRT-PCR assay or western blot analysis was performed to assess the expression level of SETDB1 mRNA or protein, respectively. siSETDB1, pCMV6-XL5-SETDB1, miR-381-3p mimic, or miR-381-3p inhibitor was transfected into cells to regulate the expression of SETDB1 or miR-381-3p. MiRNA directly interacted with SETDB1 was verified by luciferase reporter assay and RNA immunoprecipitation. CCK-8 assay, colony formation assay, flow cytometric analysis, and transwell assay were used to detect the abilities of cell proliferation, cell cycle progression and migration, respectively. Animal model of xenograft tumor was used to observe the regulatory effect of SETDB1 on tumor growth in vivo.
\end{abstract}

Results: We verified that SETDB1 mRNA level was upregulated in breast cancer tissues and cell lines, and SETDB1 depletion led to a suppression of cell proliferation, cell cycle progression and migration in vitro, as well as tumor growth in vivo. SETDB1 was verified to be a target of miR-381-3p. Moreover, miR-381-3p overexpression suppressed cell proliferation, cell cycle progression and migration, whereas SETDB1 abated miR-381-3p-mediated regulatory function on breast cancer cells.

Conclusions: This study revealed that SETDB1 knockdown might suppress breast cancer progression at least partly by miR-381-3p-related regulation, providing a novel prospect in breast cancer therapy.

Keywords: SET domain bifurcated 1, miR-381-3p, Breast cancer, Proliferation, Cell cycle progression, Migration

\section{Background}

Breast cancer, one of the most common malignancy in women, is a leading cause of cancer death all over the world [1]. Although the developments of diagnosis and therapy techniques have highly improved the survival rate of breast cancer patients, up to $30 \%$ of patients died from palindromia and metastasis after having the standard-of-care therapy [2]. Therefore, it is of importance to explore the molecular mechanism of breast cancer pathogenesis for the effective therapy.
*Correspondence: shuokunbobo@sina.com

Department of Oncology, The Affiliated Hospital of Qinghai University,

No. 29, Tongren Rd, Xining 810001, Qinghai, China
SET domain bifurcated 1 (SETDB1), also known as KMET1 or ESET, is a novel SET domain protein with histone H3 lysine 9 (H3K9)-specific methyltransferase activity which plays a pivotal role in early embryonic development [3]. It is recruited to the chromatin for silence promoters via the methyl-CpG-binding protein MBD1 [4] or some tumor inhibitor genes such as P53BP2 and RASSF1A [5]. Accumulating evidences suggest that SETDB1 might function as a novel oncogene to be involved in multiple human cancers, such as hepatocellular carcinoma [6], lung cancer [7], and sporadic cutaneous melanoma [8]. Recently, a research document demonstrated that SETDB1 was regulated by miR-7 played a critical role in the metastasis of breast cancer [9]. Moreover, the abnormal expression of SETDB1 
protein was found in human breast cancer cell lines by SILAC-based proteomic analysis [10]. Nevertheless, the molecular mechanism by which SETDB1 regulates breast cancer tumorigenesis is still unknown.

MicroRNAs (miRNAs), a type of short non-proteincoding RNAs with 20-22 nucleotides, are negative regulators of gene expression by base-pairing to the $3^{\prime}$ untranslated regions (3'-UTR) of mRNAs [11]. Following binding to partially complementary sites, miRNAs lead to a repression of translation and degradation of transcript [12]. Growing amount of evidences have suggested that miRNAs implicate in multiple physiological and developmental cellular processes, such as cell growth, differentiation, autophagy and apoptosis [13]. Dysregulation of some miRNAs has been widely acknowledged to be involved in a multitude of human cancers, including breast cancer [14]. Of these miRNAs, miR-126 was found to inhibit breast tumor proliferation and growth, and miR-335 repressed tumor metastasis [15]. Whereas, upregulated miR-10b was positively correlated with cell migration and invasion through targeting homeobox D10 (HOXD10) in breast cancer [16].

Here, we asked whether SETDB1 played a certain role partly by miRNAs regulation in breast cancer. We found that SETDB1 level was upregulated in breast cancer, and SETDB1 knockdown repressed tumor growth in vitro and in vivo. Moreover, SETDB1 was verified to be a functional target of miR-381-3p. Therefore, this study hinted that SETDB1 knockdown might repress breast cancer progression at least partly by miR-381-3p-related regulation, highlighting a novel therapeutic target for breast cancer treatment.

\section{Methods}

\section{Clinical tissues collection}

Forty five pairs of breast cancer tissues and adjacent normal breast tissues were obtained from patients who underwent radical mammectomy at the Affiliated Hospital of Qinghai University. No systemic or local treatment was performed in these patients before surgery. Tissue samples were immersed in RNAlater (Qiagen, Hilden, Germany) and snap frozen immediately, followed by stored at $-80{ }^{\circ} \mathrm{C}$ until used. Written informed consent were obtained from all patients prior to the study, and the study was approved by the Institutional Review Broad and Ethical Committee of Affiliated Hospital of Qinghai University.

\section{Cell culture}

Human mammary epithelial cell line (MCF-10A) and breast cancer cell lines (MCF-7, MDA-MB-231) that were purchased from American Type Culture Collection (ATCC, Manassas, VA, USA), were maintained in
Dulbecco's Modified Eagle Medium (DMEM, Gibco, Rockville, MD, USA) with $10 \%$ fetal bovine serum (FBS, Gibco), $1 \%$ penicillin/streptomycin (Gibco) in a humidified incubator $\left(5 \% \mathrm{CO}_{2}\right)$ at $37^{\circ} \mathrm{C}$.

\section{Cell transfection}

Small interference RNA targeting SETDB1 (siSETDB1) and the homologous negative control (siNC) were purchased from Applied Biosystems (Foster city, CA, USA). Modified miRNA mimic for hsa-miR-381-3p (miR-381-3p mimic) and its cognate negative control (miR-NC), miR-381-3p inhibitor and its negative control (inhibitor-NC), SETDB1 overexpression plasmid (pCMV6-XL5-SETDB1) were commercially synthesized by GenePhama (Shanghai, China). As previously described [17], $10 \mathrm{nM}$ of oligonucleotides or $2 \mu \mathrm{g}$ of plasmids were transfected into breast cancer cells using Lipofectamine ${ }^{\circledR}$ RNAiMAX $^{\mathrm{TM}}$ transfection reagent (Invitrogen, Waltham, MA, USA) referring to the manual of application.

\section{Quantitative real-time PCR (qRT-PCR)}

Total RNA was extracted from tissues and cells using TRIzol $^{\mathrm{TM}}$ plus RNA Purification Kit (Invitrogen) according to the manual of application. RNA quality was evaluated by an Agilent 2100 Bioanalyzer (Agilent Technologies, Santa Clara, CA, USA). Then, a total of $500 \mathrm{ng}$ RNA was reverse-transcribed into cDNA using the M-MLV reverse transcriptase (Promega, Madison, WI, USA). qRT-PCR analysis was performed in triplicates with the SsoFast ${ }^{\mathrm{TM}}$ EvaGreen Supermix (Bio-Rad, Hercules, CA, USA) on a StepOnePlus ${ }^{\text {TM }}$ Real-Time PCR System (Applied Biosystems). The expression level of SETDB1 mRNA was calculated using $2^{-\Delta \Delta C t}$ algorithm and normalized to that of GAPDH. The following primers were used: SETDB1 mRNA: 5'-GACTCTCTGAGACAACTT CCAAGGA-3' (forward) and $5^{\prime}$-CAGGGATTGAGG GAGGAACA- $3^{\prime}$ (reverse); GAPDH: 5'-TGCACCACC AACTGCTTAGC-3' (forward) and 5'-GGCATGCAC TGTGGTCATGAG-3' (reverse).

\section{Cell proliferation assay}

Cell proliferation capacity was detected by Cell Counting Kit-8 Detection Kit (CCK-8, Dojindo Molecular Technologies, Shanghai, China) referring to the protocol of manufacturer. In brief, at $0,24,48$, and $72 \mathrm{~h}$ after transfection, $10 \mu \mathrm{l}$ of CCK-8 solution was added to each well and incubated at $37{ }^{\circ} \mathrm{C}$ for $3 \mathrm{~h}$. Absorbance value at $450 \mathrm{~nm}$ was measured by a microplate reader (Bio-Rad).

\section{Colony formation assay}

Transfected cells were trypsinized into a single-cell suspension and were cultured in growth medium at $37{ }^{\circ} \mathrm{C}$ 
for 14 days to form natural colony. Then, the colonies were fixed with $4 \%$ paraformaldehyde (Sigma-Aldrich, St. Louis, MO, USA) and stained with $0.5 \%$ crystal violet (Sigma-Aldrich).

\section{Flow cytometric analysis of cell cycle progression}

Cell cycle progression was assessed by flow cytometry with Cell Cycle and Apoptosis Analysis Kit (Beyotime Institution of Biotechnology, Shanghai, China). Briefly, at $48 \mathrm{~h}$ after transfection, cells were harvested and fixed in pre-cooling $70 \%$ ethanol overnight. Following PBS-washing three times, the cells were stained with $100 \mu \mathrm{g} / \mathrm{ml}$ propidium iodide (PI) at $37^{\circ} \mathrm{C}$ for $30 \mathrm{~min}$. The evaluation of cell cycle was performed using the Modfit 3.0 software (Verity Software House, Topsham, ME, USA) on a FACScalibur flow cytometer (Becton-Dickinson, Franklin Lakes, NJ, USA).

\section{Cell migration assay}

Transfected cells were seeded on the upper chamber of an $8-\mu \mathrm{m}$ pore size Transwell (Corning, Toledo, NY, USA) to detect the cell migration ability. Upper medium was replaced by serum-free medium, while the lower chamber contained growth medium with 10\% FBS. After incubation for $24 \mathrm{~h}$, migrated cells were fixed in methanol and stained with $0.5 \%$ crystal violet (Sigma-Aldrich). At last, the number of migrated cells was counter with a microscope (Leica, Wetzlar, Germany).

\section{Luciferase reporter assay}

A fragment of SETDB1 3 -UTR containing the putative binding site of miR-381-3p, was amplified from human genomic DNA and inserted into a modified pGL3 Luciferase Reporter Vector (Promega) to construct wild SETDB1 3'-UTR report vector (SETDB1 3'-UTR-WT). To construct mutated SETDB1 $3^{\prime}$-UTR report vector (SETDB1 3'-UTR-MUT), the residues that base-pairs with miR-381-3p were mutated by site-directed mutagenesis with Q5 Site-Directed Mutagenesis Kit (New England Biolabs, Ipswich, MA, USA). $20 \mu \mathrm{g}$ of SETDB1 3'-UTR-WT or SETDB1 3'-UTR-MUT construct was cotransfected with $200 \mathrm{ng}$ of miR-381-3p mimic or miR381-3p inhibitor into MDA-MB-231 or MCF-7 cells. $48 \mathrm{~h}$ later, the luciferase activity was measured using a Lumat LB9508 luminometer (Berthold, Bad Wildbad, Germany).

\section{RNA immunoprecipitation (RIP)}

Coimmunoprecipitation (co-IP) experiment of miRNA with anti-Argonaute1 (anti-Ago1, Abcam, Cambridge, UK) was performed as previously reported [18]. Briefly, cells were transfected with miR-NC or miR-381-3p mimic for $48 \mathrm{~h}$, and were lysed with cell lysis buffer (25 mM Tris- $\mathrm{HCl}, \mathrm{pH}=7.5,150 \mathrm{mM} \mathrm{KCl}, 2 \mathrm{mM}$ EDTA,
0.5\% NP-40, $1 \mathrm{mM} \mathrm{DTT,} 100 \mathrm{U} / \mathrm{ml}$ RNasin). Then, the complex of anti-Ago1 and Protein A magnetic beads was added to cell lysates, and incubated at $4{ }^{\circ} \mathrm{C}$ overnight to get the immunoprecipitation complex. Lastly, the enrichment of SETDB1 mRNA was measured by qRT-PCR assay and anti-IgG (Abcam) was as negative control.

\section{Western blot analysis}

Protein fractions were obtained from cells using ice-cold RIPA buffer ( $50 \mathrm{mM}$ Tris- $\mathrm{HCl}, \mathrm{pH}=7.5,150 \mathrm{mM} \mathrm{NaCl}$, 1\% TritonX-100, 1 mM EDTA, $2.5 \mathrm{mM}$ sodium pyrophosphate, $1 \mathrm{mM} \beta$-glycerophosphate) supplemented with protease and phosphatase inhibitor cocktails (Roche Diagnostics, Mannheim, Germany). About $50 \mu \mathrm{g}$ of protein extractive was subjected to $10 \%$ sodium dodecyl sulfate polyacrylamide gel electrophoresis gel (10\% SDSPAGE) and transferred to polyvinyldene fluoride (PVDF) membrance (Bio-Rad). Followed by blocking with $5 \%$ non-fat milk at room temperature for $1 \mathrm{~h}$, the membrances were incubated with SETDB1 (1:2000, Abcam) and $\beta$-actin (1:5000, Abcam) antibodies at $4{ }^{\circ} \mathrm{C}$ overnight. Then, the membrances were further probed with horseradish peroxidase-conjugated secondary antibodies (1:5000, Cell Signaing Technology, Danvers, MA, USA) for $1 \mathrm{~h}$ at room temperature. All protein bands were analyzed using an Image-Pro plus 4.5 software (Media Cybernetics, Silver Spring, MD, USA).

\section{In vivo animal model}

All animals used were under an approved protocol of the Institutional Animal Care and Use Committee of Affiliated Hospital of Qinghai University. 6-8 weeks male BALB/c mice were purchased from Qinghai Research Center of Laboratory Animal (Xining, China) and were housed in a specific-pathogen-free environment. For xenograft tumors formation, $1.0 \times 10^{6} \mathrm{MCF}-7$ cells transfected with siNC or siSETDB1 were subcutaneously injected into the nude mice ( $\mathrm{n}=8$ per group). Tumor volume was determined with a caliper every 10 days. On 50 days after cell implantation, mice were euthanized and tumors were excised for weight assessment and qRT-PCR assay of SETDB1 expression.

\section{Statistical analysis}

All data were presented as mean \pm standard deviation (SD) from three independent experiments. Differences between two groups were compared using Student's $t$-test. Kaplan-Meier method was used to evaluate overall survival, and log-rank test was performed to analyze the difference in survival between two groups. $P$ value of $<0.05$ was considered statistically significant. 


\section{Results}

Upregulation of SETDB1 mRNA in breast cancer tissues and cell lines

The expression level of SETDB1 mRNA in breast cancer tissues and cell lines was firstly assessed by qRTPCR assay. As shown in Fig. 1a, the relative expression of SETDB1 mRNA was significantly elevated in breast cancer tissues compared with normal tissues. The data also revealed that SETDB1 mRNA level was higher in breast cancer cell lines than that in normal MCF-10A cells (Fig. 1b).

Subsequently, Kaplan-Meier survival assay and log-rank test using patient post-operative survival were performed to further determine the correlation between the expression level of SETDB1 mRNA and the prognosis of breast cancer patients. According to the median ratio of SETDB1 mRNA expression, the 45 breast cancer patients were classified into two groups: high SETDB1 mRNA expression group $(\mathrm{n}=24)$ and low SETDB1 mRNA expression group $(n=21)$. Kaplan-Meier survival curve presented that low SETDB1 mRNA expression group had markedly longer survival times compared to those with high SETDB1 mRNA expression group $(P<0.01$, Fig. 1c). Taken together, these data hinted that abnormal expression of SETDB1 might be associated with the progression of breast cancer.
SETDB1 knockdown inhibited proliferation, cell cycle progression and migration in breast cancer cells in vitro To explore the function of SETDB1 on breast cancer progression, loss-of-function analyses were performed by silencing the gene expression with siRNA targeting SETDB1 (siSETDB1) in vitro. Western blot assay displayed that SETDB1 protein level was drastically repressed by transfection with siSETDB1 compared with that of control in MCF-7 and MDA-MB-231 cells (Fig. 2a). Interestingly, CCK-8 and colony formation assays showed that MCF-7 and MDA-MB-231 cells in the siSETDB1 groups had lower proliferation capacity and colony formation efficiency compared to homologous control (Fig. 2b, c). Moreover, SETDB1 depletion hampered cell cycle progression through triggering the G0/G1 accumulation in MCF-7 and MDA-MB-231 cells (Fig. 2d). In cell migration assays, exogenous downregulation of SETDB1 expression remarkably repressed cell migration ability (Fig. 2e). All these results suggested that SETDB1 knockdown might be sufficient to inhibit cell proliferation, cell cycle progression and migration in breast cancer cell lines in vitro.

\section{Knockdown of SETDB1 repressed tumor growth in vivo}

Then, MCF-7 cells transfected with siSETDB1 were subcutaneously inoculated into the mice to evaluate whether SETDB1 could regulate tumor growth in vivo.

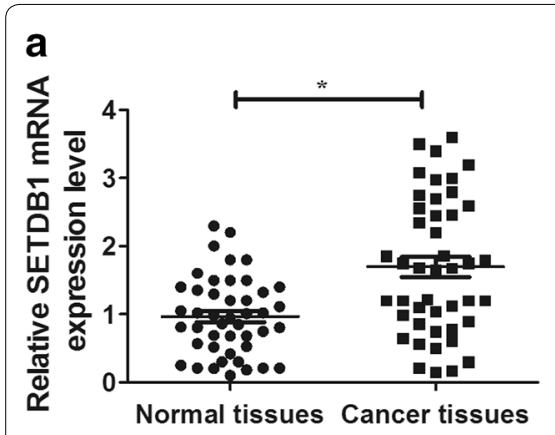

b

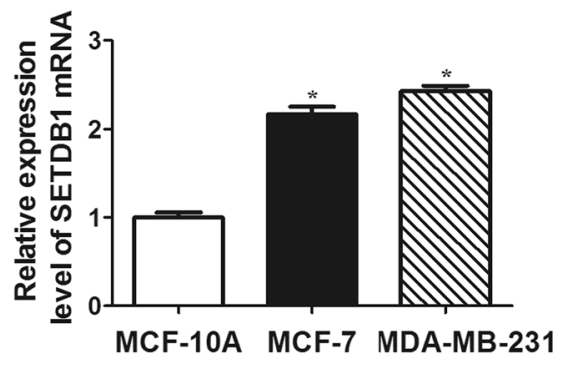

C

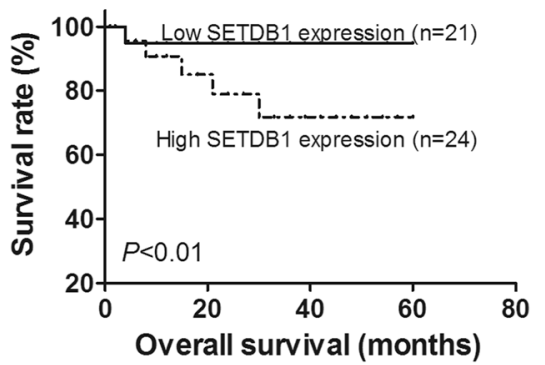

Fig. 1 SETDB1 mRNA expression was upregulated in breast cancer tissues and cell lines. a SETDB1 mRNA expression was measured in 45 pairs breast cancer tissues and adjacent normal breast tissues by qRT-PCR assay. b qRT-PCR assay of SETDB1 mRNA level in breast cancer cell lines (MCF-7, MDA-MB-231) and human mammary epithelial cell line (MCF-10A). c Kaplan-Meier survival assay and log-rank test were used to evaluate the correlation between SETDB1 mRNA level and breast cancer patient prognosis in 45 breast cancer patients in low- and high-risk groups based on SETDB1 mRNA expression. ${ }^{*} P<0.05$ vs. corresponding control

(See figure on next page.)

Fig. 2 SETDB1 knockdown suppressed proliferation, cell cycle progression and migration in MCF-7 and MDA-MB-231 cells. Western blot assay of SETDB1 expression (a), CCK-8 assay (b) and colony formation assay (c) of cell proliferation capacity, flow cytometry analysis of cell cycle progression (d), and transwell assay of cell migration ability (e) in MCF-7 and MDA-MB-231 cells transfected with siNC or siSETDB1. ${ }^{*} P<0.05$ vs. siNC 

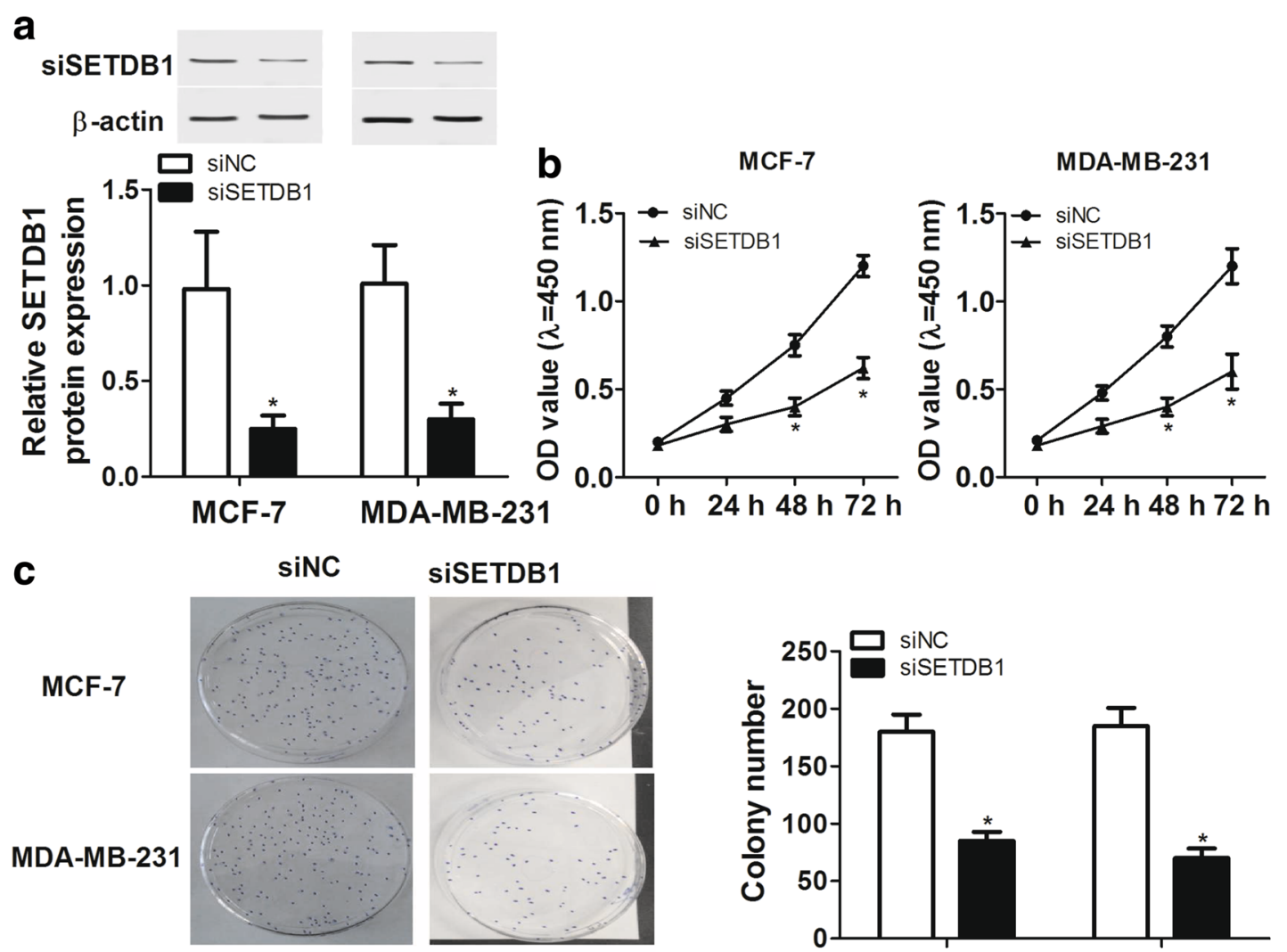
siSETDB1
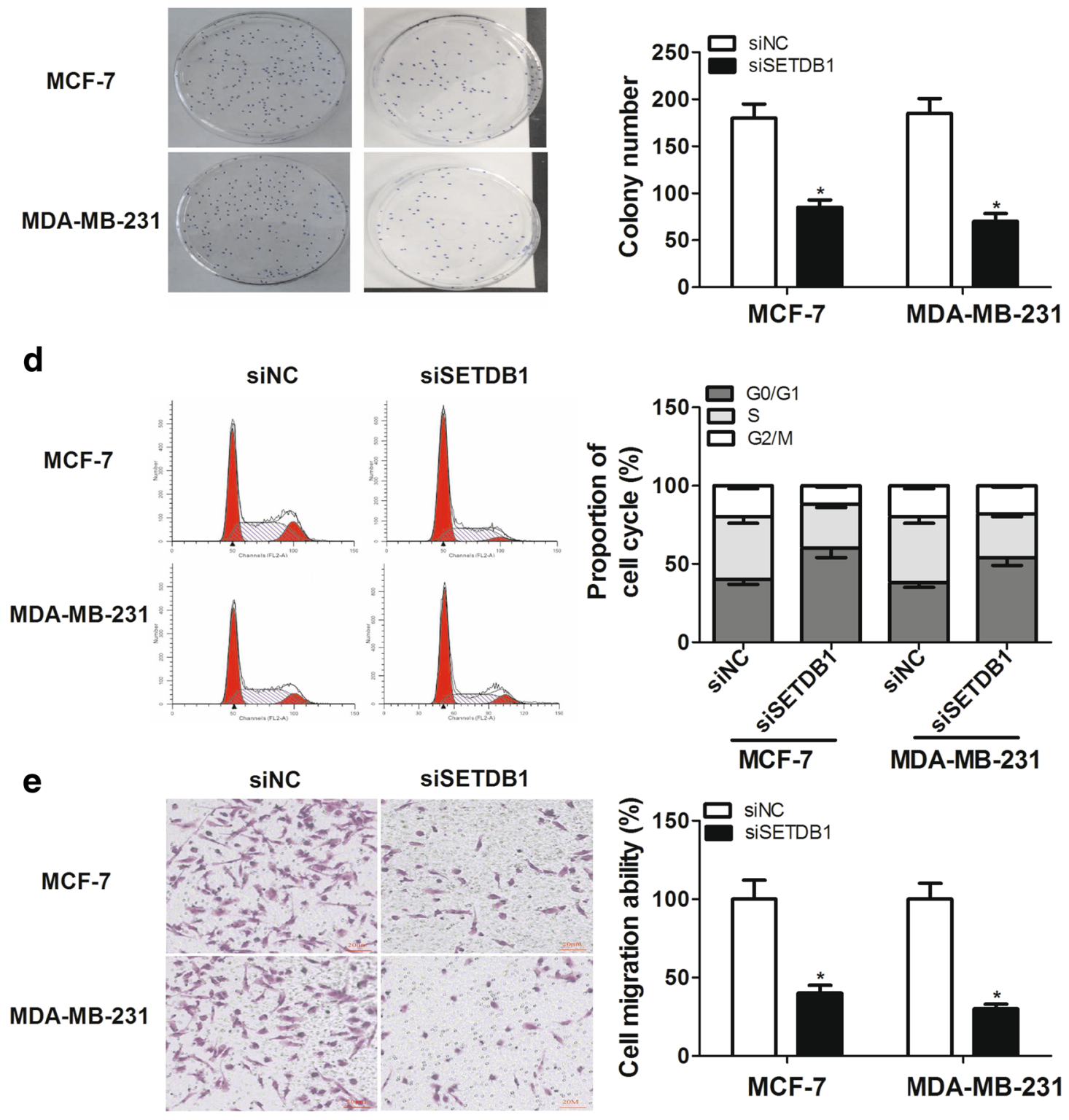
These results revealed that SETDB1 knockdown highly repressed tumor growth, presented as the decrease of tumor volume (Fig. 3a) and tumor weight (Fig. 3b, c). Moreover, as shown in Fig. 3d, SETDB1 mRNA level was significantly downregulated in tumors derived from siSETDB1-transfected cells compared to that of control. These data implied that SETDB1 knockdown might suppress tumorigenesis in vivo.

Regulation of SETDB1 by miR-381-3p in a direct interaction To further understand the molecular mechanism by which SETDB1 regulated tumor growth, online database was used to search for the miRNAs directly interacted with SETDB1 in breast cancer. Among the predicted miRNAs, miR-381-3p was chose for further research because it was confirmed to be involved in the development and progression of breast cancer [19]. The SETDB1 mRNA contained a 3'-UTR segment that was partially complementary to miR-381-3p (Fig. 4a). Subsequently, luciferase reporter assay and RNA immunoprecipitation (RIP) assay were performed to verify the correction between SETDB1 and miR-381-3p in breast cancer cells. For luciferase reporter assay, the partially sequence of SETDB1 3'-UTR was inserted into pGL3 Luciferase Reporter Vector as described previously [20]. SETDB1 3'-UTR-WT or SETDB1 3'-UTRMUT construct was cotransfected with miR-381-3p mimic or miR-381-3p inhibitor into MDA-MB-231 or MCF-7 cells. The data revealed that the luciferase activity of SETDB1 3'-UTR-WT construct was significantly

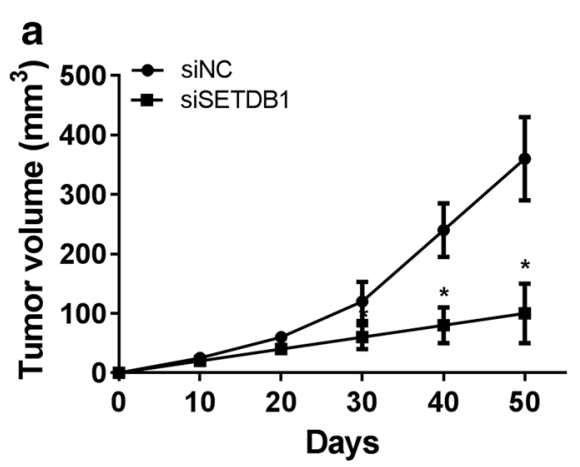

\section{b}

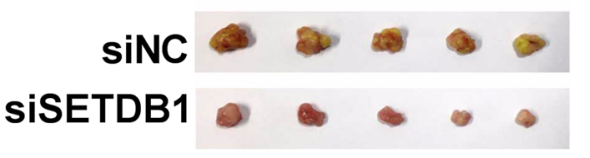

C
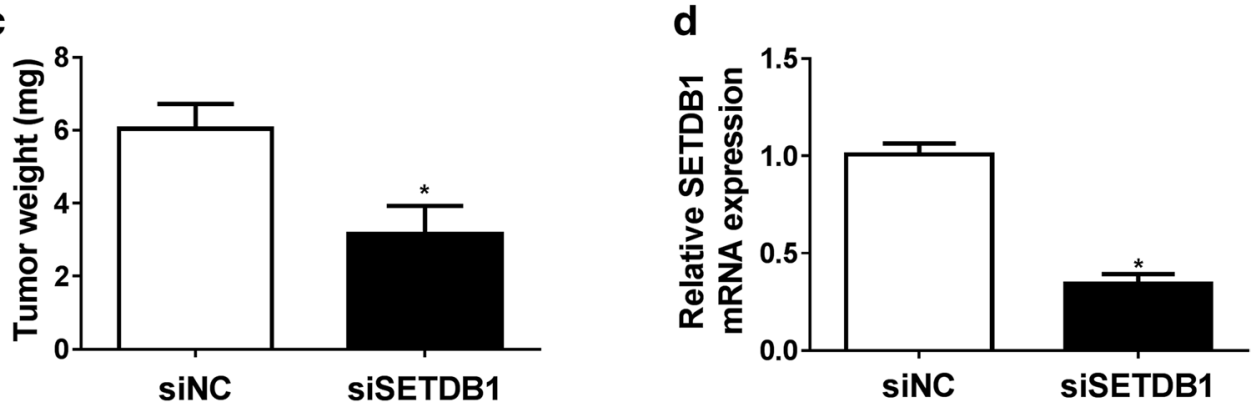

Fig. 3 SETDB1 depletion repressed tumor growth in vivo. Nude mice were subcutaneously injected with $1.0 \times 10^{6} \mathrm{MCF}-7$ cells transfected with siNC or siSETDB1. 50 days later, mice were killed and tumor masses were removed. a Tumor volume was mearsured by a caliper every 10 days. b Bright-field imaging of the xenograft tumors. c The average weight of the xenograft tumors. $\mathbf{d}$ qRT-PCR assay of SETDB1 mRNA expression in removed tumor tissues. ${ }^{*} P<0.05$ vs. siNC control

(See figure on next page.)

Fig. 4 SETDB1 was regulated by miR-381-3p in a direct interaction in breast cancer cell lines. a Schematic of potential binding sites for miR-381-3p in the SETDB1 $3^{\prime}$-UTR, the seed and the mutated sequences of potential binding sites. Luciferase reporter assays were performed to verify the interaction between miR-381-3p and SETDB1 by cotransfection with SETDB1 3'-UTR-WT or SETDB1 3'-UTR-MUT construct and miR-381-3p mimic into MDA-MB-231 cells (b) and MCF-7 cells (c), or by cotransfection with SETDB1 3'-UTR-WT or SETDB1 3'-UTR-MUT construct and miR-381-3p inhibitor into MDA-MB-231 cells (d) and MCF-7 cells (e). f RIP analysis was used to evaluate the binding between miR-381-3p and SETDB1 using anti-Ago 1 in MCF-7 and MDA-MB-231 cells transfected with miR-381-3p mimic, followed by measurement of SETDB1 mRNA by qRT-PCR assay. $\mathbf{g}$ Western blot analysis of SETDB1 protein expression in MCF-7 and MDA-MB-231 cells transfected with miR-381-3p mimic or miR-381-3p inhibitor. ${ }^{*} P<0.05$ vs. respective negative control 
a

hsa-miR-381-3p

SETDB1 WT 3'UTR SETDB1MUT 3'UTR
3' UGUCUCUCGAACGGGAACAUA U 5'

| | | || |

5'...UCUACUAUCUCCAGUUUGUAUU...3'

5'...UCUACUAUCUCCAGUAACAUAU...3' b

MDA-MB-231

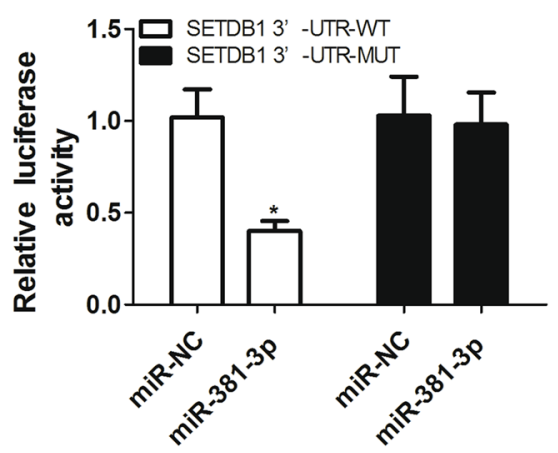

d

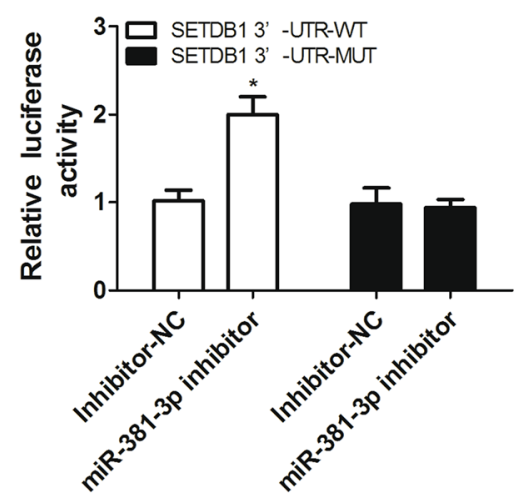

f

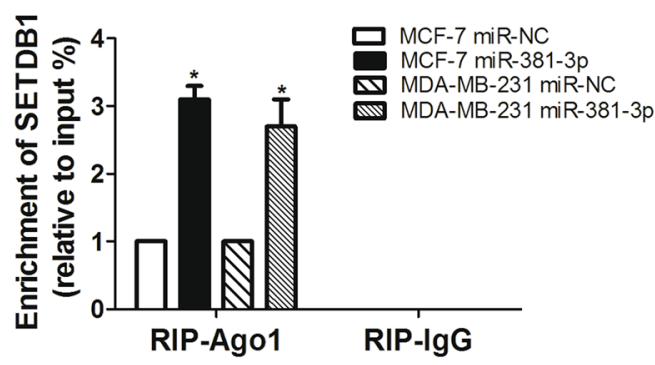

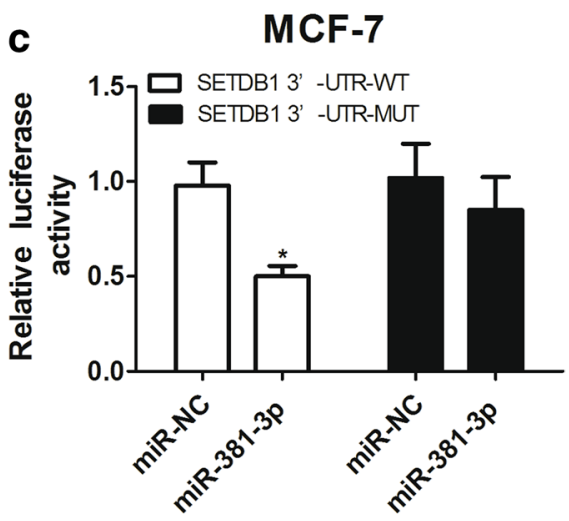
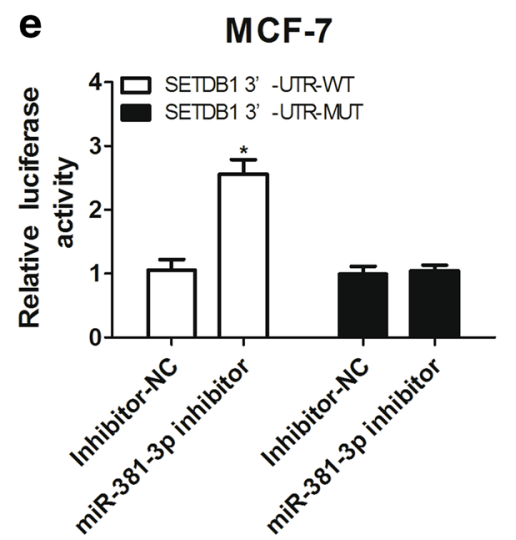

g

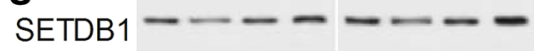

$\beta$-actin -----
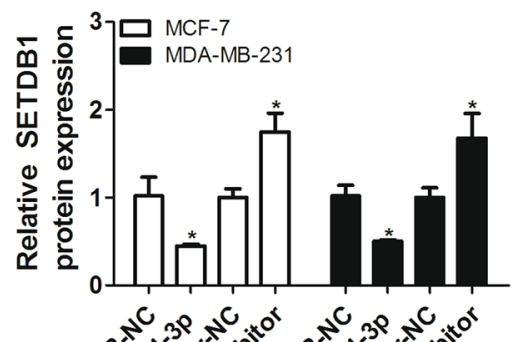
reduced by miR-381-3p overexpression (Fig. 4b, c), while it was highly enhanced by miR-381-3p depletion (Fig. 4d, e) in MDA-MB-231 and MCF-7 cells. Whereas, little change was found in the luciferase activity of SETDB1 3'-UTR-MUT construct in response to the alteration of miR-381-3p expression (Fig. 4b-e). RIP analysis showed that SETDB1 mRNA was specifically recruited to the miRNP complexes isolated with anti-Ago1 following miR-381-3p transfection in MDA-MB-231 and MCF-7 cells (Fig. 4f). Additionally, we further detected whether SETDB1 expression was regulated by miR-381-3p in MDA-MB-231 and MCF-7 cells. Western blot analysis showed that compared with corresponding counterparts, SETDB1 expression was markedly weakened by transfection with miR-381-3p mimic, while it was remarkably promoted following miR-381-3p inhibitor introduction (Fig. 4g). All these results indicated that SETDB1 was a direct target of miR-381-3p.

\section{Restoration of SETDB1 expression abrogated the regulatory function of miR-381-3p in breast cancer cell lines}

Then, miR-381-3p mimic was transfected into MCF-7 and MDA-MB-231 cells to assess the function of miR381-3p on breast cancer progression. As shown in Fig. 5a, SETDB1 expression was markedly inhibited by transfection with miR-381-3p mimic. Functional analysis revealed that miR-381-3p overexpression led to the suppression of cell proliferation capacity (Fig. 5b, c), colony formation ability (Fig. 5d), and cell cycle progression (Fig. 5e), as well as cell migration ability (Fig. 5f) in MCF-7 and MDA-MB-231 cells. Together, these data suggested that miR-381-3p might suppress cell proliferation, cell cycle progression and migration in breast cancer cell lines.

Further, to explore whether the suppression function of miR-381-3p was mediated by SETDB1, MCF-7 and MDA-MB-231 cells were cotransfected with miR-381-3p mimic and SETDB1 overexpression vector (pCMV6XL5-SETDB1). The data illustrated that miR-381-3pinduced the suppression of SETDB1 expression was drastically abated by cotransfection with pCMV6-XL5SETDB1 (Fig. 5a). Moreover, the restoration of SETDB1 expression remarkably abrogated miR-381-3p-mediated inhibition effect on cell proliferation capacity, colony formation ability, cell cycle progression and migration ability in MCF-7 and MDA-MB-231 cells (Fig. 5b-f). All these findings hinted that the restoration of SETDB1 expression undermined miR-381-3p-mediated retardation on proliferation, cell cycle progression and migration in breast cancer cell lines.

\section{Discussion}

Histone lysine methylation has been reported to be implicated in transcriptional activation or suppression, which was of increasing interest owing to its involved in the neoplastic transformation [21]. H3K9 methylation was reported to play a vital role in multiple human cancer [22]. SETDB1, located at chromosome 1q21, is a member of H3K9 methylation catalyzed by histone lysine methyltransferases [23]. Over the past few years, SETDB1 has been widely considered as an oncogene playing a critical role in many human cancers. It was previously reported that SETDB1 protein level was upregulated in human melanomas tissues and it enhanced the formation of melanoma in a zebrafish model [24]. Silence of SETDB1 was found to inhibit lung tumor growth in vitro and in vivo, while it upregulation promoted the tumor invasiveness, highlighting its role as a novel therapeutic target [25]. SETDB1 also was reported to be overexpressed in hepatocellular carcinoma (HCC), which inactivation repressed the growth of $\mathrm{HCC}$ cell lines through regulating p53 methylation [26]. In addition to the cancers mentioned above, SETDB1 was proposed as an oncogene in prostate cancer [27], gliomas [28] and colorectal cancer [29]. In this study, SETDB1 level was verified to be elevated in breast cancer tissues and cell lines, which was consistent with the finding of Zhang et al. [9]. From a functional standpoint, SETDB1 knockdown inhibited breast tumor growth in vitro and in vivo. All these data suggested that SETDB1 knockdown might suppress breast cancer progression.

MiRNAs are attractive candidates as upstream regulators of tumor progression because they have been demonstrated to repress a set of target genes expression in a post-transcriptional way [30]. For instance, miR-135 and miR-203 inhibited breast tumor growth and metastasis in vitro and in vivo by targeting runx2 [31]. MiR-155 was found to accelerate tumor angiogenesis by directly suppressing von Hippel-Lindau (VHL) expression in triplenegative breast cancer [32]. It was reported that miR-621 overexpression promoted breast cancer chemosensitivity through targeting FBXO11 [33]. Moreover, miR-21 enhanced epithelial-to-mesenchymal transition (EMT) by inhibiting PTEN protein expression in breast cancer [34]. PTPRN2, MERTK, TNC and SOX4 were identified to be targets of miR-335 [15], AIB1 and CCND1 were the targets of miR-17-5p [35], and H-RAS and HMGA2 were verified as targets of let-7 [36] in breast cancer.

Then, software algorithms were performed to search for the miRNAs directly interacted with SETDB1 in breast cancer. Among the predicted miRNAs, miR381-3p was chose for further research owing to its involvement in the progression of multiple human cancers. For example, in oral squamous cell carcinoma, 


\section{a}

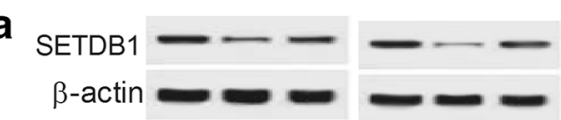

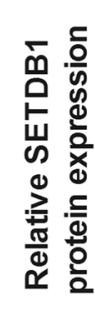

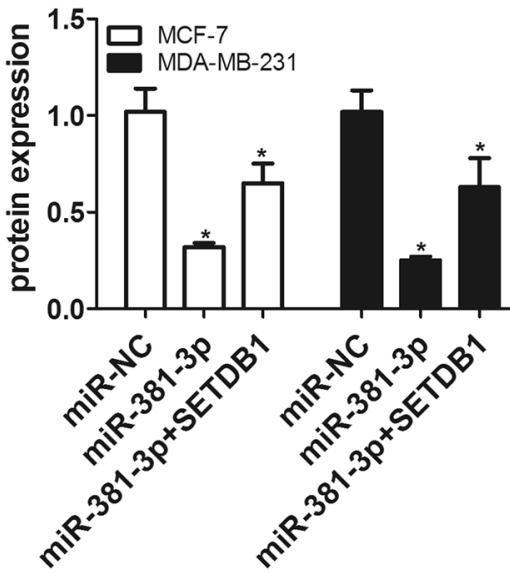

C

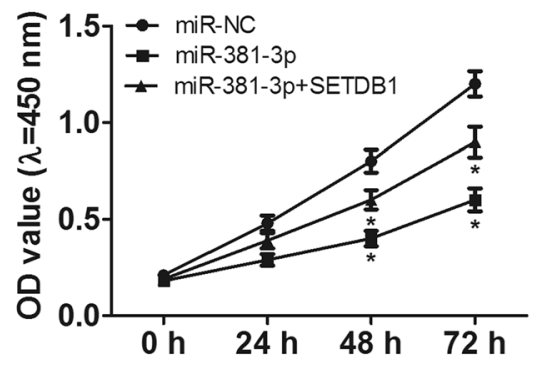

e
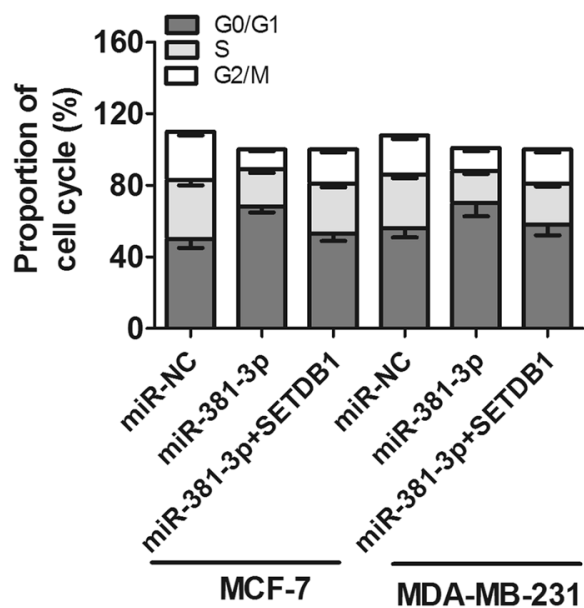

b

\section{MCF-7}

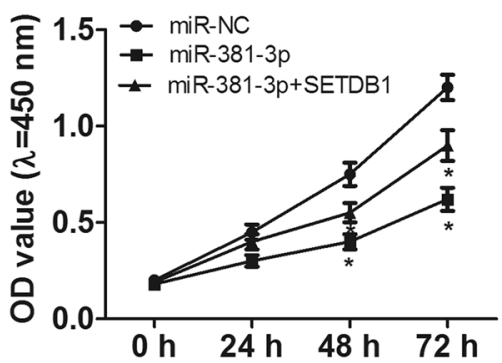

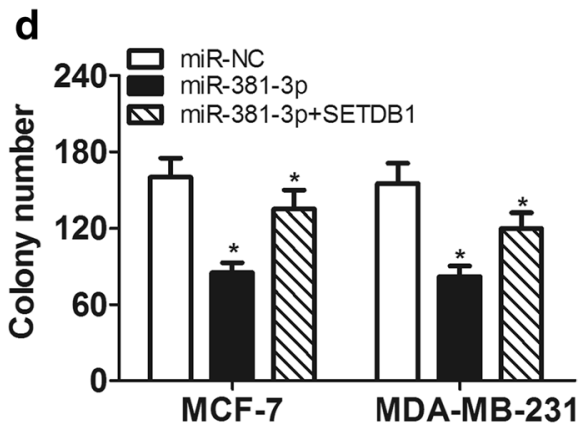

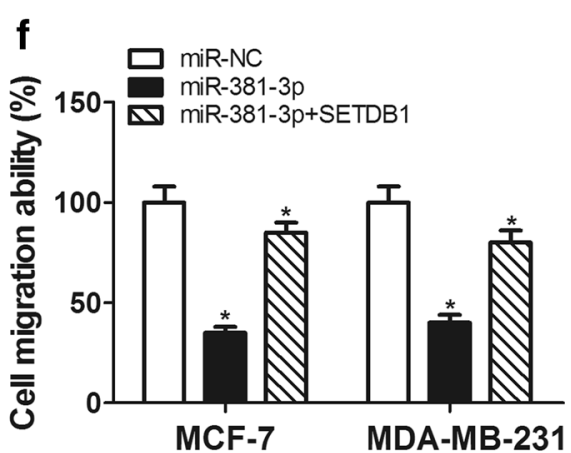

Fig. 5 SETDB1 abrogated miR-381-3p-mediated inhibition on proliferation, cell cycle progression and migration in breast cancer cell lines. a Western blot analysis of SETDB1 expression, $\mathbf{b}, \mathbf{c}$ CCK-8 assay and $\mathbf{d}$ colony formation assay of cell proliferation capacity, e flow cytometry analysis of cell cycle progression, and $\mathbf{f}$ transwell assay of cell migration ability in MCF-7 and MDA-MB-231 cells transfected with miR-381-3p mimic or miR-381-3p mimic + pCMV6-XL5-SETDB1. ${ }^{*} P<0.05$ vs. corresponding control (miR-NC or miR-381-3p mimic) 
miR-381-3p suppressed cell proliferation and cell cycle progression while enhanced apoptosis through directly targeting fibroblast growth factor receptor 2 (FGFR2) [37]. In non-small cell lung cancer, miR-381 led to a suppression of tumor growth and chemoresistance by direct downregulation of differentiation 1 (ID1) [38]. Additionally, a recent document reported that miR-381 suppressed proliferation, EMT and metastasis of breast cancer cells through targeting CXCR4 [19]. In the present study, SETDB1 was verified to be a functional target of miR-381-3p in breast cancer cells. Consistent with the findings of Xue et al. [19], miR-381-3p was manifested to suppress breast cancer cells proliferation, cell cycle progression and migration. Moreover, miR381-3p-mediated regulatory function was abrogated by the restoration of SETDB1 expression.

\section{Conclusions}

In conclusion, our study demonstrated that SETDB1 was upregulated in breast cancer and SETDB1 knockdown suppressed breast cancer progression at least partly by miR-381-3p-related regulation, highlighting SETDB1 as a novel biomarker for breast cancer therapy.

\begin{abstract}
Abbreviations
SETDB1: SET domain bifurcated 1; H3K9: H3 lysine 9; 3'-UTR: 3' untranslated regions; HOXD10: homeobox D10; siSETDB1: small interference RNA targeting SETDB1; qRT-PCR: quantitative real-time PCR; RIP: RNA immunoprecipitation; CO-IP: coimmunoprecipitation; PVDF: polyvinyldene fluoride.
\end{abstract}

\section{Authors' contributions}

This work was conceived and designed by MW, BF and QG. The experiments were carried out by YL, RC and NL. The manuscript was prepared by MW, YD and YL. All authors read and approved the final manuscript.

\section{Competing interests}

The authors declare that they have no competing interests.

\section{Availability of data and materials}

All data generated and analyzed during this study are included in this published article and its additional information files. The gene expression dataset used is available on request.

\section{Consent for publication}

Not applicable.

\section{Ethics approval and consent to participate}

All studies involving animal subjects were approved by the institutional animal care and use committees at the Affiliated Hospital of Qinghai University.

\section{Funding and acknowledgements}

This work was supported by grants from the National Natural Science Foundation of China (81760730) and the Scientific Research Project Funds of Qinghai Department (2016-ZJ-785).

\section{Publisher's Note}

Springer Nature remains neutral with regard to jurisdictional claims in published maps and institutional affiliations.
Received: 13 July 2018 Accepted: 3 October 2018

Published online: 11 October 2018

\section{References}

1. DeSantis C, Ma J, Bryan L, Jemal A. Breast cancer statistics, 2013. CA Cancer J Clin. 2014;64(1):52-62.

2. Miller KD, Siegel RL, Lin CC, Mariotto AB, Kramer JL, Rowland JH, et al. Cancer treatment and survivorship statistics, 2016. CA Cancer J Clin. 2016;66(4):271-89.

3. Matsui T, Leung D, Miyashita H, Maksakova IA, Miyachi H, Kimura H, et al. Proviral silencing in embryonic stem cells requires the histone methyltransferase ESET. Nature. 2010:464(7290):927-31.

4. Sarraf SA, Stancheva I. Methyl-CpG binding protein MBD1 couples histone $\mathrm{H} 3$ methylation at lysine 9 by SETDB1 to DNA replication and chromatin assembly. Mol Cell. 2004;15(4):595-605.

5. Li H, Rauch T, Chen ZX, Szabo PE, Riggs AD, Pfeifer GP. The histone methyltransferase SETDB1 and the DNA methyltransferase DNMT3A interact directly and localize to promoters silenced in cancer cells. J Biol Chem. 2006;281(28):19489-500.

6. Wong CM, Wei L, Law CT, Ho DW, Tsang FH, Au SL, et al. Up-regulation of histone methyltransferase SETDB1 by multiple mechanisms in hepatocellular carcinoma promotes cancer metastasis. Hepatology. 2016;63(2):474-87.

7. Wu PC, Lu JW, Yang JY, Lin IH, Ou DL, Lin YH, et al. H3K9 histone methyltransferase, KMT1E/SETDB1, cooperates with the SMAD2/3 pathway to suppress lung cancer metastasis. Cancer Res. 2014;74(24):7333-43.

8. Kostaki M, Manona AD, Stavraka I, Korkolopoulou P, Levidou G, Trigka EA, et al. High-frequency p16(INK) (4A) promoter methylation is associated with histone methyltransferase SETDB1 expression in sporadic cutaneous melanoma. Exp Dermatol. 2014;23(5):332-8.

9. Zhang H, Cai K, Wang J, Wang X, Cheng K, Shi F, et al. MiR-7, inhibited indirectly by lincRNA HOTAIR, directly inhibits SETDB1 and reverses the EMT of breast cancer stem cells by downregulating the STAT3 pathway. Stem Cells. 2014;32(11):2858-68.

10. Cuomo A, Moretti S, Minucci S, Bonaldi T. SILAC-based proteomic analysis to dissect the "histone modification signature" of human breast cancer cells. Amino Acids. 2011;41(2):387-99.

11. van Kouwenhove $M$, Kedde $M$, Agami R. MicroRNA regulation by RNA-binding proteins and its implications for cancer. Nat Rev Cancer. 2011:11(9):644-56.

12. Croce CM. Causes and consequences of microRNA dysregulation in cancer. Nat Rev Genet. 2009;10(10):704-14.

13. Finnerty JR, Wang WX, Hebert SS, Wilfred BR, Mao G, Nelson PT. The miR-15/107 group of microRNA genes: evolutionary biology, cellular functions, and roles in human diseases. J Mol Biol. 2010;402(3):491-509.

14. Iorio MV, Ferracin M, Liu CG, Veronese A, Spizzo R, Sabbioni S, et al. MicroRNA gene expression deregulation in human breast cancer. Cancer Res. 2005;65(16):7065-70.

15. Tavazoie SF, Alarcon C, Oskarsson T, Padua D, Wang Q, Bos PD, et al. Endogenous human microRNAs that suppress breast cancer metastasis. Nature. 2008:451(7175):147-52.

16. Ma L, Teruya-Feldstein J, Weinberg RA. Tumour invasion and metastasis initiated by microRNA-10b in breast cancer. Nature. 2007;449(7163):682-8.

17. Cao M, Seike M, Soeno C, Mizutani H, Kitamura K, Minegishi Y, et al. MiR23a regulates TGF-beta-induced epithelial-mesenchymal transition by targeting E-cadherin in lung cancer cells. Int J Oncol. 2012;41(3):869-75.

18. Meister G, Landthaler M, Peters L, Chen PY, Urlaub H, Luhrmann R, et al. Identification of novel argonaute-associated proteins. Curr Biol. 2005:15(23):2149-55.

19. Xue Y, Xu W, Zhao W, Wang W, Zhang D, Wu P. miR-381 inhibited breast cancer cells proliferation, epithelial-to-mesenchymal transition and metastasis by targeting CXCR4. Biomed Pharmacother. 2017;86:426-33.

20. Clement T, Salone V, Rederstorff M. Dual luciferase gene reporter assays to study miRNA function. Methods Mol Biol. 2015;1296:187-98.

21. Martin C, Zhang Y. The diverse functions of histone lysine methylation. Nat Rev Mol Cell Biol. 2005;6(11):838-49. 
22. Kondo Y, Shen L, Ahmed S, Boumber Y, Sekido Y, Haddad BR, et al. Downregulation of histone $\mathrm{H} 3$ lysine 9 methyltransferase G9a induces centrosome disruption and chromosome instability in cancer cells. PLoS ONE. 2008;3(4):e2037.

23. Karimi MM, Goyal P, Maksakova IA, Bilenky M, Leung D, Tang JX, et al. DNA methylation and SETDB1/H3K9me3 regulate predominantly distinct sets of genes, retroelements, and chimeric transcripts in mESCs. Cell Stem Cell. 2011;8(6):676-87.

24. Ceol CJ, Houvras Y, Jane-Valbuena J, Bilodeau S, Orlando DA, Battisti V, et al. The histone methyltransferase SETDB1 is recurrently amplified in melanoma and accelerates its onset. Nature. 2011;471(7339):513-7.

25. Rodriguez-Paredes M, de Paz AM, Simo-Riudalbas L, Sayols S, Moutinho C, Moran S, et al. Gene amplification of the histone methyltransferase SETDB1 contributes to human lung tumorigenesis. Oncogene. 2014;33(21):2807-13.

26. Fei Q, Shang K, Zhang J, Chuai S, Kong D, Zhou T, et al. Histone methyltransferase SETDB1 regulates liver cancer cell growth through methylation of p53. Nat Commun. 2015;6:8651.

27. Sun Y, Wei M, Ren SC, Chen R, Xu WD, Wang FB, et al. Histone methyltransferase SETDB1 is required for prostate cancer cell proliferation, migration and invasion. Asian J Androl. 2014;16(2):319-24.

28. Spyropoulou A, Gargalionis A, Dalagiorgou G, Adamopoulos C, Papavassiliou KA, Lea RW, et al. Role of histone lysine methyltransferases SUV39H1 and SETDB1 in gliomagenesis: modulation of cell proliferation, migration, and colony formation. Neuromolecular Med. 2014;16(1):70-82.

29. Kim HA, Koo BK, Cho JH, Kim YY, Seong J, Chang HJ, et al. Notch1 counteracts WNT/beta-catenin signaling through chromatin modification in colorectal cancer. J Clin Invest. 2012;122(9):3248-59.

30. Lim LP, Lau NC, Garrett-Engele P, Grimson A, Schelter JM, Castle J, et al Microarray analysis shows that some microRNAs downregulate large numbers of target mRNAs. Nature. 2005;433(7027):769-73.
31. Taipaleenmaki H, Browne G, Akech J, Zustin J, van Wijnen AJ, Stein $J$, et al. Targeting of Runx2 by miR-135 and miR-203 impairs progression of breast cancer and metastatic bone disease. Cancer Res. 2015;75(7):1433-44.

32. Kong W, He L, Richards EJ, Challa S, Xu CX, Permuth-Wey J, et al. Upregulation of miRNA-155 promotes tumour angiogenesis by targeting $\mathrm{VHL}$ and is associated with poor prognosis and triple-negative breast cancer. Oncogene. 2014;33(6):679-89.

33. Xue J, Chi Y, Chen Y, Huang S, Ye X, Niu J, et al. MiRNA-621 sensitizes breast cancer to chemotherapy by suppressing FBXO11 and enhancing p53 activity. Oncogene. 2016;35(4):448-58.

34. Wu ZH, Tao ZH, Zhang J, Li T, Ni C, Xie J, et al. MiRNA-21 induces epithelial to mesenchymal transition and gemcitabine resistance via the PTEN/AKT pathway in breast cancer. Tumour Biol. 2016;37(6):7245-54.

35. Hossain A, Kuo MT, Saunders GF. Mir-17-5p regulates breast cancer cell proliferation by inhibiting translation of AIB1 mRNA. Mol Cell Biol. 2006;26(21):8191-201.

36. Yu F, Yao H, Zhu P, Zhang X, Pan Q, Gong C, et al. let-7 regulates self renewal and tumorigenicity of breast cancer cells. Cell. 2007:131(6):1109-23.

37. Yang X, Ruan H, Hu X, Cao A, Song L. miR-381-3p suppresses the proliferation of oral squamous cell carcinoma cells by directly targeting FGFR2. Am J Cancer Res. 2017;7(4):913-22.

38. Huang RS, Zheng YL, Zhao J, Chun X. microRNA-381 suppresses the growth and increases cisplatin sensitivity in non-small cell lung cancer cells through inhibition of nuclear factor-kappaB signaling. Biomed Pharmacother. 2018;98:538-44.
Ready to submit your research? Choose BMC and benefit from:

- fast, convenient online submission

- thorough peer review by experienced researchers in your field

- rapid publication on acceptance

- support for research data, including large and complex data types

- gold Open Access which fosters wider collaboration and increased citations

- maximum visibility for your research: over 100M website views per year

At BMC, research is always in progress.

Learn more biomedcentral.com/submissions 